\title{
Using Mobile Sensing Technology for Capturing People Mobility Information
}

\author{
Chenwei Song \\ Institute of Industrial \\ Science \\ The University of Tokyo \\ Tokyo, Japan \\ songchenwei@mcl.iis.u- \\ tokyo.ac.jp
}

\author{
Masaki Ito \\ Institute of Industrial \\ Science \\ The University of Tokyo \\ Tokyo, Japan \\ mito@iis.u-tokyo.ac.jp
}

\author{
Yuuki Nishiyama \\ Institute of Industrial \\ Science \\ The University of Tokyo \\ Tokyo, Japan \\ yuukin@iis.u-tokyo.ac.jp
}

\author{
Kaoru Sezaki \\ Institute of Industrial \\ Science \\ The University of Tokyo \\ Tokyo, Japan \\ sezaki@iis.u-tokyo.ac.jp
}

\begin{abstract}
The detection and analysis of human crowds have been widely used from urban design and traffic management to disaster evacuation and mobility prediction. Currently, several common methods of crowd flow detection have different performances in terms of accuracy, cost and scope of application. One of the main reasons for the difference is that the technology or equipment used to detect crowd flows are different. For example, focus on accuracy, use the camera to record in real-time, and analyze the flow of people with the relevant algorithms. Or pay more attention to the scope of application, using the GPS information uploaded by the user when using the map service to obtain the direction and speed information of the crowd. In this paper, we propose a clientserver system based on Bluetooth scanning to obtain crowd information. Our proposed system has the advantages of low cost and location flexibility. The system can detect any area without pre-deployed, as long as there is a sufficient number of users involved.
\end{abstract}

\section{CCS CONCEPTS}

- Information systems $\rightarrow$ Sensor networks

\section{KEYWORDS}

Human mobility, Crowd detection, Bluetooth

\section{ACM Reference format:}

Chenwei Song, Masaki Ito, Yuuki Nishiyama and Kaoru Sezaki. 2019. Using Mobile Sensing Technology for Capturing People Mobility Information. In 3rd ACM SIGSPATIAL International Workshop on Prediction of Human Mobility (PredictGIS 2019), November 5, 2019, Chicago, IL, USA, 8 pages. https://doi.org/10.1145/3356995.3364541

Permission to make digital or hard copies of all or part of this work for personal or classroom use is granted without fee provided that copies are not made or distributed for profit or commercial advantage and that copies bear this notice and the full citation on the first page. Copyrights for components of this work owned by others than ACM must be honored. Abstracting with credit is permitted. To copy otherwise, or republish, to post on servers or to redistribute to lists, requires prior specific permission and/or a fee. Request permissions from Permissions@acm.org.

PredictGIS'19, November 5, 2019, Chicago, IL, USA

(C) 2019 Association for Computing Machinery.

ACM ISBN 978-1-4503-6964-0/19/11\$15.00

https://doi.org/10.1145/3356995.3364541

\section{INTRODUCTION}

A crowd is a deformable group of people occupying a particular area. By detecting people crowd and capturing the context information within a specified area, we can analyze and even predict the mobility of crowds, such as people's number, direction or speed [1]. Crowd understanding and predicting have a massive impact on several applications including surveillance and security, situation awareness, crowd management, public space design, etc. For example, the government can decide how to widen existing roads to alleviate traffic congestion based on the location information of high-density populations [2]. Besides, merchants can decide to place the advertising labels where can attract the most customers according to the walking track of the crowd on the commercial street. In the real-time environment, once a sudden increase of people in a certain area is detected, staff can immediately make evacuation work to avoid injury caused by crowding. On the other hand, users in a disaster area can select a road with a low people density according to the real-time information reported by the human crowd detection to avoid secondary damage caused by congestion [3].

Because the equipment and methods used to collect the raw data are different, the existing approaches have different performance in terms of cost, accuracy, and the scope of application. For example, the method of cameras has a high-precision performance in acquiring and analyzing crowd information, but it is not costeffective because it requires to deploy multiple cameras. On the other hand, utilizing GPS-related information uploaded by users when using the map service, the deployment scope of this method can be more flexible and wider. However, due to the inability of the GPS signal to reach the inside buildings, there are certain restrictions on the scope of application.

In this paper, we propose a crowd mobility information system based on Bluetooth. The purpose is to capture the direction and an approximate number of the crowds in a high-density population area or in a sudden disaster, so as to provide early warning for further avoidance or evacuation planning. It does not need to deploy devices such as cameras or Radio Frequency Identification (RFID) tag in advance and only needs enough users to carry 
smartphones with Internet access, which can obtain the crowd information in a certain area. We conducted several experiments in the actual environment to verify this method can accurately obtain changes of a crowd.

The rest of the paper is organized as follows. Section 2 describes the related works by other researchers. Section 3 gives details of the system structure and design. Section 4 shows the experiments that we conducted and the evaluation of the results. Section 5 is the future works. Section 6 gives the conclusion.

\section{RELATED WORKS}

Detecting the crowds to obtain counting number and mobility data is important for today's urban life. According to existing methods for collecting crowd information, we can divide the methods into two groups by different types of raw data: the image data methods and the non-image data methods. For example, using cameras is a typical method which analyzes image data. The raw data obtained by it is images of the video, then utilize human recognition technology to get the crowd information in the image. About the non-image data methods, the raw data of this kind include the sending time, location coordinates, speed, etc. of a device. How the data of the devices are linked to the information of the crowds becomes the key.

Image data methods, such as using closed-circuit television (CCTV) cameras to capture image data, find the crowd area from the image data and convert it into crowd information. Image recognition technology and equipment that can capture highresolution images to make the method highly accurate [9]. But this method relies on the image collecting equipment, so the method is costly. At the same time, the method will cause problems such as occlusion, insufficient light, inflexibility due to the position where the camera is installed. Therefore, it is more suitable for the government to monitor traffic flow and safety, etc.

In the non-image data methods, the GPS-based method has been published by many map service companies [10]. When using the map service applications, the user can get the crowd information from the server, at the same time, his phone as a participating sensor uploads information. This method mainly relies on the user's mobile phone, especially the GPS chip. It does not require complicated image information conversion process. However, the GPS signal cannot reach indoors, therefore, it cannot be applied to every place.

Wi-Fi is a recently popular indoor detection method instead of GPS. By analyzing the change of the Wi-Fi signal between multiple access points (APs) or analyzing the Wi-Fi connection data collected by the AP, we can know the flow of people in that area [11]. This method can be used in any place where an AP is deployed. In other words, it is not restricted by indoor and outdoor. However, due to the need to deploy APs, this method is more suitable for indoor detection. In the method of analyzing signals, it is also necessary to do a large number of signal samples in advance for reference, so that the method of analyzing signals is relatively complicated.

A Bluetooth method is similar to a device-based method of Wi-Fi. But because the sender and requester of Bluetooth can be converted to each other. There is no need for additional devices like APs, just make sure that the proportion of people carrying Bluetooth devices in the crowd is enough [12]. Although the effective range of Bluetooth is very short, its low cost and wide usage make this method be applied to almost anywhere.

The original purpose of Bluetooth was to meet the need for smallscale data transmission at short range [16]. So, Bluetooth has features like short communication distance, low speed and low cost. This makes Bluetooth very suitable for some special scenes.

In $[4,5]$, they installed multiple Bluetooth receiving devices in one room. When a person carrying a Bluetooth device enters the room, each receiver can obtain a Received Signal Strength Indicator (RSSI) value to display the distance to the user. Through the trilateration algorithm [5], the user's location in the room can be finally obtained. This method can be used as an alternative to GPS localization indoors. Compared to the Wi-Fi localization method, Bluetooth is cheaper and easier to install.

In [6], they proposed to build a Bluetooth Ad Hoc Network, CrowdBlueNet, to collect data from individual smartphones to support crowd management. The network is completely based on Bluetooth devices, so even if the cellular and wireless networks are not accessible, the network will still work, which means that as long as the user is inside the Bluetooth network zone, the crowd information can be obtained. The users can avoid entering areas with high density of people and causing damage.

In $[7,8]$, they developed a bus query application based on the crowdsourcing model, using Bluetooth as a crowd counting device and location conversion trigger. Let the users' smartphones provide the number of passengers and traffic conditions instead of the equipment that will be installed on the bus to achieve these proposes. Therefore, reducing the cost of investigating bus operations by using Bluetooth of users' smartphones.

As the researches we have listed above, most of the application scenarios of Bluetooth are to use the low cost and flexibility of Bluetooth to replace the original high cost and non-movable methods.

\section{METHODOLOGY}

For existing methods, there are several common problems.

1. The range of each detection device is limited, and it is necessary to continuously add devices in order to expand the range, resulting in high cost.

2. Installing the device or device in a certain area in advance to enable this method for human flow capture.

3. Some methods are impossible to capture crowd information anywhere due to the limitations of the device. 
4. Many existing methods will fail if there is an emergency such as a power outage.

In this paper, we propose a client-server-service-based system that provides users with real-time crowd information. Our system is designed based on the following two points.

1. No additional devices are required other than users' smartphones and the server.

2. The users are also sensors that provide information about surrounding Bluetooth devices.

Our goal is to implement a crowd information system built by the user's smartphone. Since the devices in the system only utilize the user's smartphones, there is no high-cost problem. There is no need to spend time deploying the device in advance, only the user needs to start Bluetooth scanning of the smartphone in the area and upload the information. In the emergency, using the Bluetooth LAN mentioned in [6], users can still use Bluetooth to share crowd information in the area without being affected by the power cut.

\subsection{System Overview}

According to statistics, in 2018, Bluetooth device shipments have reached 3.9 billion, of which smartphones, tablets and other mobile devices have reached 2.05 billion [13]. This proves that Bluetooth is everywhere in people's lives. Bluetooth has two main communication protocol technologies, Basic Rate/Enhanced Data Rate (BR/EDR) and Bluetooth Low Energy (BLE). Due to the low power consumption of BLE and the official announcement that $97 \%$ of Bluetooth chips will contain BLE mode in 2022, we decided to use BLE mode to scan. In addition, if a disaster occurs such as an earthquake or fire, there will likely be power off. At this time, common methods such as cameras or Wi-Fi routers will fail due to the power cut. In [6], the Bluetooth Ad Hoc Network, CrowdBlueNet, is able to use in such an emergency. The network is completely based on Bluetooth devices, which means that as long as the user is inside the Bluetooth network zone, the crowd information can be shared in this local area network.

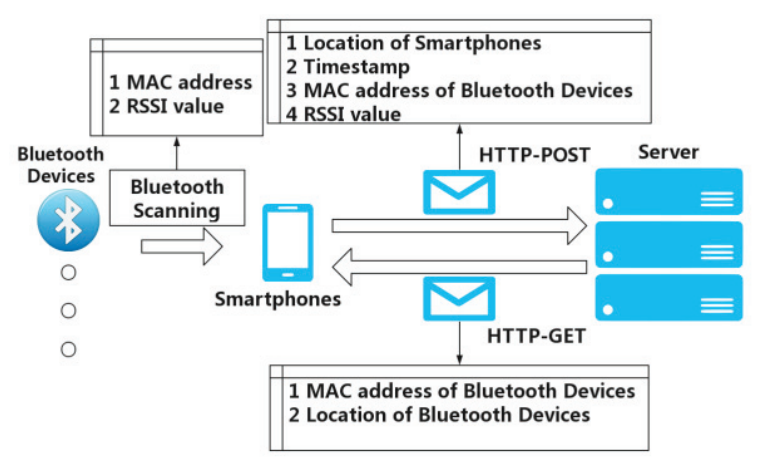

Figure 1: Structure of the System
As shown in Figure 1, the structure of the system is a client-server model. Each smartphone with our app will become a client node. They will scan and upload the crowd information while graphically displaying the data obtained from the server to the user [14]. The server receives and analyzes the information from each client node, then it returns the crowd data to each client node.

The workflow of the entire system is as follows. A user can receive the crowd information by carrying a smartphone with our application installed. The application automatically collects surrounding crowd information every 10 seconds. Then send the data to the server through the Hypertext Transfer Protocol (HTTP) method. The server receives the data from smartphones and stores the information in the database. After analysis, the server sends crowd information inside that area back to the smartphones. The smartphones display the crowd information to the users through the graphic interface of our application.

\subsection{Roles in the System}

We define the following roles to make our system easier to understand.

The user: After the users installed our application on their smartphones, they can check the crowd information in their area through the application. At the same time, their smartphones become crowd sensors, sending the crowd information surrounding them to our server through the network at regular intervals. The rest of this paper will use the user to refer to the people with smartphones installed our application.

The Bluetooth device: Bluetooth devices are the devices detected by the smartphones through Bluetooth scanning. It could be any Bluetooth device that can be discovered, such as mobile phones, watches, headphones, etc.

The server: The server is the computer we set to provide the crowd information for the smartphones. After processing the information sent by the smartphone, the server sends the result back the smartphone, such as people crowd's density, mobility and location.

\subsection{Data Collection and Analysis Method}

When the application on the smartphone starts working, it will scan the surrounding Bluetooth devices in BLE mode, then upload the device information to the server every 10 seconds. The message includes the timestamp, the location of the smartphone, and the RSSI value and the Media Access Control (MAC) Address of the scanned Bluetooth.

RSSI value: It is a measurement of the power present in a received radio signal. In this paper, it stands for the strength of the Bluetooth devices' signal scanned by users' smartphones. RSSI can be used to approximate distance between the scanned Bluetooth device and the smartphone. If we have the location of users' smartphones and the RSSI value of the Bluetooth devices 
scanned by the users, we can get the approximate location of the Bluetooth devices [19].

MAC address: A MAC address of a device is a unique identifier assigned to a network interface controller (NIC). For communications within a network segment, it is used as a network address for most IEEE 802 network technologies, including Ethernet, Wi-Fi, and Bluetooth. Like the license plate, in theory, every Bluetooth device has a unique MAC address. Therefore, we can use it to distinguish different Bluetooth devices. We can estimate the number of people in that area by the number of Bluetooth devices. Or we can predict the trend of the crowd through the change in the number of Bluetooth devices.

Location: If we can capture the accurate location of the Bluetooth device like the GPS method, the crowd mobility obtained from that will be highly reliable. But if we want to get more data from Bluetooth device through Bluetooth, in addition to Bluetooth scanning, it needs a Bluetooth connection. However, the Bluetooth connection will be more power-consuming and only one-to-one connection. Directly transmitting location information via Bluetooth connection does not work. On the other hand, in the common wireless positioning methods, they require multiple anchor points to get the signal of the measured point at the same time $[4,5,18]$. Through experiments, we find that it is rare for multiple smartphones to discover a Bluetooth device at the same time. Through measurement, we know that in an environment with a large population, the Bluetooth signal from a general mobile device can transmit no more than 20 meters. The main purpose of this paper is to grasp the general mobility information of the crowd, rather than precise positioning. This level of error is still acceptable. We will define the location of the Bluetooth device scanned by the smartphone as the location of the smartphone. In other words, we mainly use the GPS data of the smartphone to determine the location of the Bluetooth device.

After collecting the crowd information from each user, we need to further analyze the data on the server to provide useful information for the users. Each piece of data is stored by the format including the timestamp, mac address, geographic location and RSSI value. The specific meaning is that at a certain moment, a certain location, a Bluetooth device with a certain mac address is found. In order to make it the easiest and most intuitive way for users to get information about the crowd in the area, we decided to use the heat map and direction arrows to display the data analyzed by the server [14].

Heat map: A heat map is a graphical representation of data where the individual values contained in a matrix are represented as colors. We define a ten-meter-long, ten-meter-wide area as a grid point, and such multiple grid points form a grid map. By counting different MAC addresses in the same grid point at one time window, the number of the Bluetooth devices appearing in the grid point area can be obtained. Finally, using color gradients to represent the different densities of the number of people.

Direction arrow: The direction arrow can intuitively display a certain mobile node's displacement information including the starting position, ending position and moving direction. The MAC address is used to distinguish between different Bluetooth devices. If one MAC address is captured at different time points, we can get the movement track of the device [15].

\section{EXPERIMENTS}

\subsection{Effective Distance of the Bluetooth Signal}

The Bluetooth signal strength is reflected by the RSSI value, which is a negative number. The closer the distance is to the Bluetooth device, the closer the value is to zero.

The RSSI value can be converted to the distance value by the following formula.

$$
R S S I=-\left(10 n \log _{10} d+A\right)
$$

$A$ is the RSSI value when the equipment is 1 meter apart from the receiver.

$n$ is the signal transmission constant, and it is relevant to signal transmission environment.

$d$ is the distance we want to know.

After the test, we found that as the distance between the smartphone and the Bluetooth device becomes longer, the fluctuation of the RSSI signal becomes larger, and the difference between the distance converted from the formula and the actual distance becomes larger. Therefore, we need to confirm the effective sending range of Bluetooth devices which often be used in our daily lives. We conducted experiments both indoors and outdoors, setting a smartphone and a Bluetooth headset as the receiver and sender separately. Both of them support Bluetooth 5.0 , which means they can work in BLE mode. We tested the range at 5-meter intervals starting from 0 meters up to 30 meters. At each range, we measured the RSSI value. Table 1 shows the average result of this experiment.

Table 1: Result of Range Test

\begin{tabular}{rrr}
\hline Distance in meters & RSSI (Indoor) & RSSI (Outdoor) \\
\hline 0 & -40 & -40 \\
\hline 5 & -53 & -57 \\
\hline 10 & -69 & -75 \\
\hline 15 & -75 & -80 \\
\hline 20 & -97 & -99 \\
\hline 25 & -99 & -101 \\
\hline 30 & -101 & -103 \\
\hline
\end{tabular}

According to the results of the above table, we can conclude that although the Bluetooth signal can still be detected at around 30 meters, it is difficult to distinguish the distance of Bluetooth devices through the RSSI value when it is more than 20 meters. Therefore, in the following experiments, we set the software acceptable threshold to -75 in an indoor environment and -80 in 
an outdoor environment. In other words, the farthest Bluetooth device that the mobile phone can detect is 15 meters.

\subsection{Detection of Number Change in the Crowd}

In the area where the disaster occurs, there will be a significant change in the number of people around the time of the disaster, and most of them will increase dramatically [17]. Even under normal circumstances, the information of the crowd density in the designated area can also allow the users to avoid high-density areas, thereby avoiding congestion and even disaster caused by people flow. Therefore, if the number of people flows in the area or the changing trend of people flow can be obtained in real-time to capture or even predict the movement of people, it helps evacuate people or avoid congestion disasters.

In order to verify the feasibility of Bluetooth detection, we conducted the experiments in 4 different scenarios.

1. The classroom $(20 \mathrm{~m} \times 10 \mathrm{~m})$ where the professor had a lecture.

2. The metro compartment $(18 \mathrm{~m} \times 3 \mathrm{~m})$ with a random number of people getting on and off at each station.

3. The street with the low pedestrian flow

4. The crowded crossroad during the rush hour

In each experiment, we conducted a 30-minute data collection and collected a set of data every minute. Every time we collect data, we scan the Bluetooth device within a radius of 15 meters with a smartphone installed our application. At the same time take a 360degree photo and manually calculate the number of people within 15 meters.

In the experiments, the number of Bluetooth devices recorded by the smartphone may contain fixed devices, such as Bluetoothenabled printers, iBeacon, etc. However, only by recorded data from the smartphone, it is difficult to distinguish and delete fixed devices. As shown in Table 2, the value, 'Device/Per person' should be larger than the actual. The accuracy of directly converting the number of Bluetooth devices to the number of adult streams will be affected.

As shown in Figure 2, the changing trend of the number of people is very accurately reflected by the number changing of Bluetooth devices. Especially, at the time when arriving at a station, when the green light is on, etc., the number of Bluetooth devices changes rapidly as the number of people changes.

Through the experiments, we know that the number of fixed Bluetooth devices will affect the accuracy of reflecting people flow number by the number of Bluetooth devices. But, the trend of the number of Bluetooth devices can accurately reflect the trend of changes in the number of people.

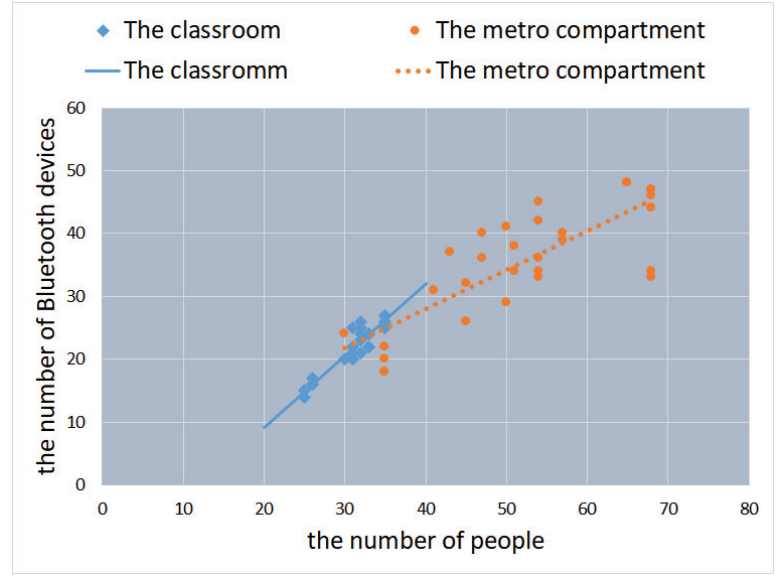

(a) Indoor Experiments

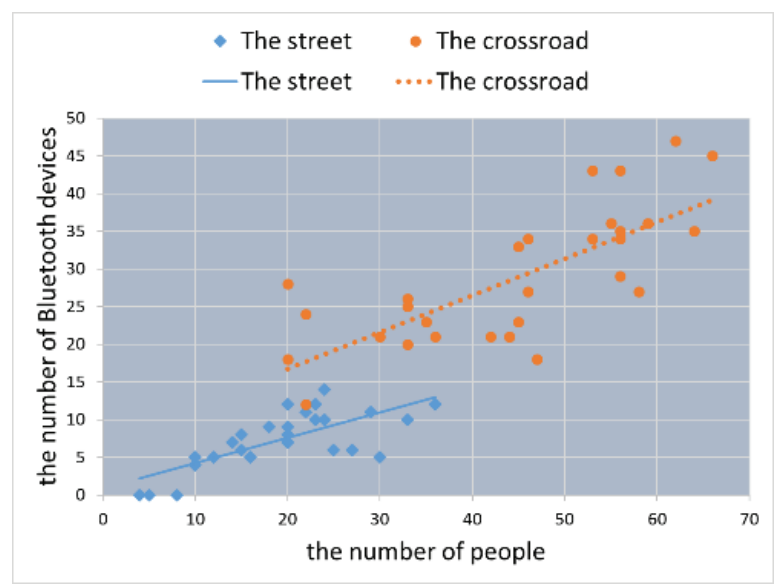

(b) Outdoor Experiments

Figure 2: Scanning Result

Table 2: Correlation Between Bluetooth Devices and People

\begin{tabular}{lrr}
\hline & $\begin{array}{l}\text { Device/Per } \\
\text { person }\end{array}$ & \multicolumn{2}{l}{$\begin{array}{l}\text { Pearson Correlation } \\
\text { Coefficient }\end{array}$} \\
\hline The classroom & 1.1442 & 0.9463 \\
\hline The metro & 0.6182 & 0.8047 \\
\hline The street & 0.3363 & 0.7483 \\
\hline The crossroad & 0.4867 & 0.7416 \\
\hline
\end{tabular}

\subsection{Analysis of Actual Use Situation}

After the feasibility test of Bluetooth scanning, we need to do a further test about the actual use situation. The previous experiments were all done by a single smartphone, verifying the feasibility of Bluetooth as an effective way to get information about people flows. In the next experiment, we want to verify the feasibility of the entire system in actual use, including the case 
where multiple smartphones are used at the same time, the actual display of the analyzed data.

Table 3: Environmental Parameters of the Experiments

\begin{tabular}{lrrr}
\hline & Route 1 & Route 2 & \multicolumn{2}{c}{ Route 3 } \\
\hline Time $(\mathrm{min})$ & 15 & 15 & 20 \\
\hline Area $\left(\mathrm{m}^{2}\right)$ & 95048.32 & 37726.35 & 36723.47 \\
\hline $\begin{array}{l}\text { Length } \\
(\mathrm{km})\end{array}$ & 1.78 & 1.12 & 1.14 \\
\hline Overlapping & $20 \%$ & $54 \%$ & $60 \%$ \\
\hline
\end{tabular}

We selected the test area near the train station in Shimokitazawa, Tokyo. We asked 5 participants to walk around the station, each holding a smartphone installed our application. The overlapping in Table 3 refers to the percentage of route repetitions of the 5 people.

Since the site is outdoors, we set the RSSI acceptance threshold to -80 , which means that the farthest Bluetooth device detected by the smartphone is at 15 meters. For the location information of the smartphone, we directly use the GPS on the smartphone to obtain. The application on the smartphone will send the surrounding flow information to the server every 10 seconds and display the analyzed data sent from the server. That means the server will receive 6 records per minute from one smartphone. Because there are 5 smartphones, the previous two experiments recorded 450 times respectively, and the third experiment recorded 600 times. We consider Bluetooth devices that have been detected more than 6 times by smartphones as an effective mobility point information. Bluetooth devices that are only detected once are classified as a suspicious point. By the amount of displacement of the same detected device between different times, we can determine if it is a fixed device. But we can't classify devices that are only detected once.

Table 4: Analysis of Data Validity

\begin{tabular}{lccc}
\hline & Route 1 & Route 2 & Route 3 \\
\hline $\begin{array}{l}\text { Detected } \\
\text { devices }\end{array}$ & 726 & 549 & 1122 \\
\hline $\begin{array}{l}\text { Detected } \\
\text { more } \\
\text { than six } \\
\text { times }\end{array}$ & $217(29.9 \%)$ & $357(65.0 \%)$ & $639(57.0 \%)$ \\
\hline $\begin{array}{l}\text { Detected } \\
\text { only once }\end{array}$ & $290(40.0 \%)$ & $126(23.0 \%)$ & $381(34.0 \%)$ \\
\hline
\end{tabular}

From Table 4 we can see that the route overlapping rate will affect the proportion of suspicious points and valid points. Smartphones with a low overlapping rate can obtain device information only within its Bluetooth scan range (In this experiment, it is 15 meters.). The low overlapping rate also means that in most cases, multiple smartphones are actually in their own range, scanning and uploading crowd information as a single node. Once the Bluetooth device is out of the measurement range of the smartphone, the device can no longer be captured. However, if the overlapping rate rises, even if the Bluetooth device is outside the scanning range of one smartphone, it is possible to be captured again by other smartphones in the overlapping range, the detection of the device remains valid.

According to the principle of the trilateration algorithm [5], to obtain location information of the measured point, we need at least three points with known location information and their respective distances to the measured point. In our system, if three or more smartphones can detect the same Bluetooth device at the same time, by converting the RSSI value into the distance value, we can obtain the specific location information of the Bluetooth device.

We analyzed the feasibility of Bluetooth devices position. From Table 5 we concluded that in the experiment, the proportion of device that can be located is too low. The device position in our system is based on the number of smartphones that can detect the same Bluetooth device at the same time, which means that the proportion of participants meeting each other needs to be high. The column, 'Times that testers met each other' of Table 5 shows that the five participants are mostly distributed in different locations in the test area, that is the main reasons for the low proportion of devices that can be located. Therefore, in the case where the user overlap rate is low in the region, the system is not suitable for accurately positioning the device. The location of the smartphone is used instead of the position of the Bluetooth device surrounding the smartphone.

Table 5: Analysis of Data Correlation

\begin{tabular}{lccc}
\hline & Route 1 & Route 2 & Route 3 \\
\hline Record times & 450 & 450 & 600 \\
\hline $\begin{array}{l}\text { Times that } \\
\text { testers met } \\
\text { each other }\end{array}$ & $26(5.7 \%)$ & $56(12.4 \%)$ & $64(7.1 \%)$ \\
\hline $\begin{array}{l}\text { Record by } \\
\text { more than } \\
\text { three } \\
\text { smartphones } \\
\text { at the same } \\
\text { time }\end{array}$ & $59(13.1 \%)$ & $126(28 \%)$ & $167(18.6 \%)$ \\
\end{tabular}

When the actual use test was performed, each tester's smartphone displays the data as shown in Figure 3. The application will get the crowd data of last 10 seconds from the server every 2 seconds. After removing the duplicate data with the MAC address, it displays the information directly on the smartphone. As shown in Figure 3, Each red icon indicates that a smartphone uploaded information in the past 10 seconds. The number above the red icon represents the number of surrounding Bluetooth devices 
scanned by the smartphone. The method visualizes the geographic information and the number of Bluetooth devices, allowing users to roughly understand the crowd density of the area through the number of Bluetooth devices in the area.

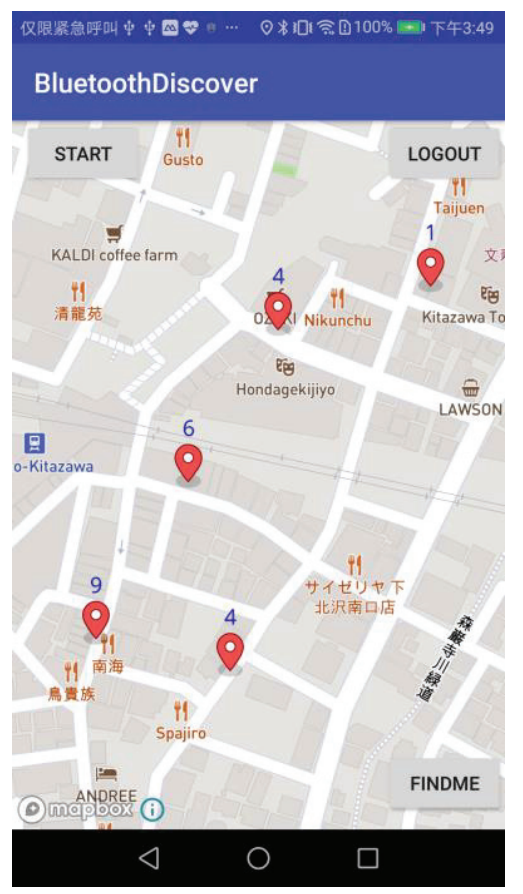

Figure 3: Screenshot of Real-time Mode

\section{FUTURE WORKS}

In order to make it easier for users to understand the change of flow density and the direction of flow in a certain area, we also added the heat map mode and direction arrow mode.

Heat map mode: If there are many users in an area, too many icons and numbers will affect the user to read the crowd information. Obviously, the method of icons and numbers is not suitable for the situation of many users in the area. The heat map can effectively display the crowd density of the entire area with different colors. We define a ten-meter-long, ten-meter-wide area as a grid point, and such multiple grid points form a grid map. By counting different MAC addresses in the same grid point at one time window, the number of the Bluetooth devices appearing in the grid point area can be obtained. We divide the crowd data in the past 10 minutes into 101 -minute time windows and average the flow density of 10 time windows. Finally, using color gradients to represent the different densities of the number of people.

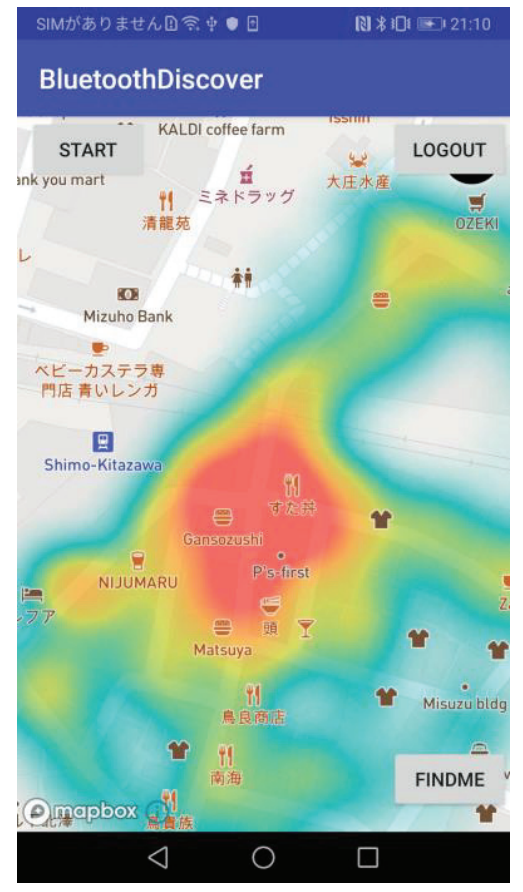

Figure 4: Screenshot of Heat Map Mode

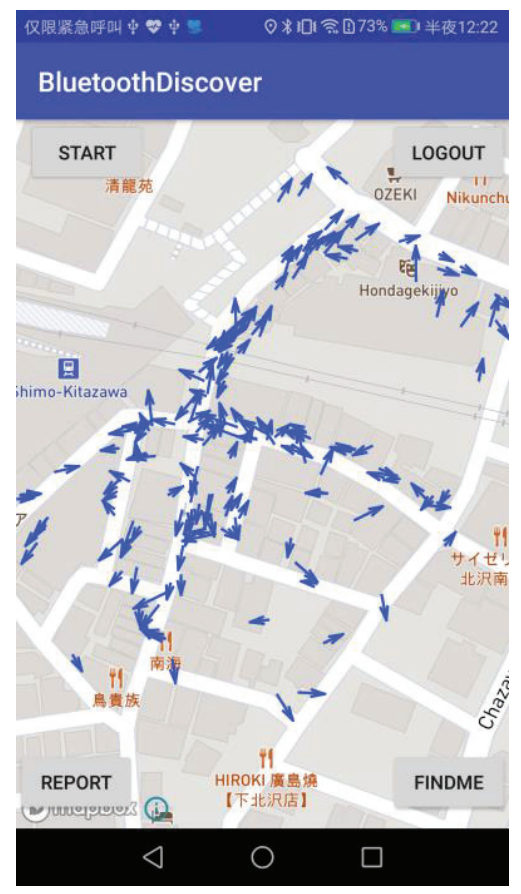

Figure 5: Screenshot of Direction Arrow Mode

Direction arrow: The direction arrow can intuitively display a certain mobile node's displacement information including the starting position, ending position and moving direction. The direction arrow mode also uses the same time-division method as the heat map mode. Analyze data from the past 10 minutes as a 
period, where each minute is divided into a time window. In one minute, the average of the geographic locations of devices with the same MAC address, resulting in a point. Then connect the points obtained in multiple time windows with arrow-lines. This creates a map of the direction arrows. Through the arrows, we can roughly get the movement of the crowd.

In the future, we will conduct experiments in other practical locations to verify whether these visualization modes accurately display the density changes of people flow and the direction of crowds.

\section{CONCLUSION}

In this paper, we propose a crowd information system based on Bluetooth scanning. It does not require the addition of equipment such as cameras or routers, so it has the advantage of low cost and flexibility, which also solves the problem of existing common methods mentioned in Section 3. We verified its accuracy and feasibility through the experiments. The experiments show that the change in the number of Bluetooth devices in a certain area does reflect the change in the number of people in the area. If the users have a higher path overlap rate in the same area, the more accurate data the system provides. In other words, the more users participate, the better the result will obtain.

As future work, we need to test in different crowd situations to verify the system's capture of different crowds changes. At the same time, compare the results with the existing methods to display the accuracy of our proposed system.

\section{ACKNOWLEDGEMENT}

This work was supported by JST CREST Grant Number JPMJCR1411-14531840, Japan.

\section{REFERENCES}

[1] Haghani, M., \& Sarvi, M. (2018). Crowd behaviour and motion: Empirical methods. Transportation research part B: methodological, 107, 253-294.

[2] Sagl, G., Resch, B., \& Blaschke, T. (2015). Contextual sensing: Integrating contextual information with human and technical geo-sensor information for smart cities. Sensors, 15(7), 17013-17035.

[3] Do, T. M. T., \& Gatica-Perez, D. (2012, September). Contextual conditional models for smartphone-based human mobility prediction. In Proceedings of the 2012 ACM conference on ubiquitous computing (pp. 163-172). ACM.

[4] Wang, Y., Yang, X., Zhao, Y., Liu, Y., \& Cuthbert, L. (2013, January). Bluetooth positioning using RSSI and triangulation methods. In 2013 IEEE 10th Consumer Communications and Networking Conference (CCNC) (pp. 837-842). IEEE

[5] Rida, M. E., Liu, F., Jadi, Y., Algawhari, A. A. A., \& Askourih, A. (2015, April). Indoor location position based on bluetooth signal strength. In 2015 2nd International Conference on Information Science and Control Engineering (pp. 769-773). IEEE.

[6] Liu, S., Du, J., Yang, X., Li, R., Liu, H., \& Sha, K. (2016, August). CrowdBlueNet: Maximizing Crowd Data Collection Using Bluetooth Ad Hoc Networks. In International Conference on Wireless Algorithms, Systems, and Applications (pp. 356-366). Springer, Cham.

[7] Aihara, K., Bin, P., \& Imura, H. (2019, July). On the Relationship Between Accuracy of Bus Position Estimated by Crowdsourcing and Participation Density. In International Conference on Human-Computer Interaction (pp. 101-112). Springer, Cham.

[8] Cianciulli, D., Canfora, G., \& Zimeo, E. (2017). Beacon-based context-aware architecture for crowd sensing public transportation scheduling and user habits. Procedia Computer Science, 109, 1110-1115.
[9] Baqui, M., \& Löhner, R. (2017, May). Real-time crowd safety and comfort management from CCTV images. In Real-Time Image and Video Processing 2017 (Vol. 10223, p. 1022304). International Society for Optics and Photonics.

[10] Blanke, U., Tröster, G., Franke, T., \& Lukowicz, P. (2014, April). Capturing crowd dynamics at large scale events using participatory gps-localization. In 2014 IEEE Ninth International Conference on Intelligent Sensors, Sensor Networks and Information Processing (ISSNIP) (pp. 1-7). IEEE.

[11] Depatla, S., \& Mostofi, Y. (2018, June). Passive Crowd Speed Estimation and Head Counting Using WiFi. In 2018 15th Annual IEEE International Conference on Sensing, Communication, and Networking (SECON) (pp. 1-9). IEEE.

[12] Basalamah, A. (2016). Sensing the crowds using bluetooth low energy tags. IEEE access, 4, 4225-4233.

[13] Bluetooth, S. I. G (2018) Bluetooth 5 Core Specification, Version 5, 06 December 2016.

[14] Sekimoto, Y., Sudo, A., Kashiyama, T., Seto, T., Hayashi, H., Asahara, A., .. \& Nishiyama, S. (2016, September). Real-time people movement estimation in large disasters from several kinds of mobile phone data. In Proceedings of the 2016 ACM International Joint Conference on Pervasive and Ubiquitous Computing: Adjunct (pp. 1426-1434). ACM.

[15] Vu, L., Nguyen, P., Nahrstedt, K., \& Richerzhagen, B. (2015). Characterizing and modeling people movement from mobile phone sensing traces. Pervasive and Mobile Computing, 17, 220-235.

[16] Kountouriotis, V., Thomopoulos, S. C., \& Papelis, Y. (2014). An agent-based crowd behaviour model for real time crowd behaviour simulation. Pattern Recognition Letters, 44, 30-38.

[17] Krausz, B., \& Bauckhage, C. (2012). Loveparade 2010: Automatic video analysis of a crowd disaster. Computer Vision and Image Understanding, 116(3), 307-319.

[18] Ji, M., Kim, J., Jeon, J., \& Cho, Y. (2015, July). Analysis of positioning accuracy corresponding to the number of BLE beacons in indoor positioning system. In 2015 17th International Conference on Advanced Communication Technology (ICACT) (pp. 92-95). IEEE.

[19] Li, G., Geng, E., Ye, Z., Xu, Y., Lin, J., \& Pang, Y. (2018). Indoor positioning algorithm based on the improved RSSI distance model. Sensors, 18(9), 2820 